\title{
Optimization Design of a Carbon Fibre Prosthetic Foot for Amputee
}

\author{
Huy-Tuan Pham ${ }^{1 *}$, Thanh-Vu Phan' ${ }^{1}$ and Van-Trinh Mai ${ }^{2}$ \\ ${ }^{1}$ Faculty of Mechanical Engineering, Ho Chi Minh City University of Technology and \\ Education, Vietnam \\ ${ }^{2}$ Orthopaedic and Rehabilitation Centre, Ministry of Labour - Invalids and Social \\ Affairs, Ho Chi Minh city, Vietnam \\ *Corresponding Author: Huy-Tuan Pham, Faculty of Mechanical Engineering, Ho \\ Chi Minh City University of Technology and Education, Vietnam.
}

Received: August 31, 2020

Published: September 21, 2020

(C) All rights are reserved by Huy-Tuan

Pham., et al.

\begin{abstract}
Compliant prosthetic foot is a popular term for all passive prosthesis that are currently preferable by amputee due to their advantage of energy saving, light weight, simple structure and easier usage over the powered counterparts. The key characteristic of this device replies on the elastic deformation of the structure to support its functions. In order to fulfil this property, design and material of the devices are the two most concerns. This paper describes an optimization design method for a prosthetic foot made from carbon fibre. Finite element analyses are carried out to evaluate the mechanical behaviours of the design obtained by the optimization procedure. Prototypes of the device are also fabricated and tested. The characteristics of the optimum prosthetic foot predicted by theory are not only quantitatively verified by experiments but also qualitatively surveyed by trials from the amputee. Results show that the fabricated prototype is capable of bending and compression to store elastic energy and release it to assist in forward propulsion of the body. These characteristics make it more versatile and reduce the impact force on residual limbs.
\end{abstract}

Keywords: Prosthetic; Compliant Mechanism; Composites; Carbon Fibre; Optimization

\section{Abbreviations}

ESAR: The Energy Storage and Return Foot; CTM: Constant-Torque Mechanism; CFP: Carbon-Fibre Prosthesis

\section{Introduction}

Function loss of amputees due to residual limbs usually induces an abnormal gait. Therefore, a prosthesis is expected to fully replicate the functions of the natural organ it replaces. While active prosthetics with an internal power sources are still in the stage of development [1-3], passive devices are widely used in the market. Normally, disable people were prescribed solid ankle cushioned heels, which mostly focused on reducing impact loading at heel strike, but stores and releases very little elastic energy [4]. The energy storage and return (ESAR) foot is an alternative which has the potential to help improve gait [5]. Despite the variety of available commercial products in the market, it is still intricate to make them as flexible as the intact feet.

Lee., et al. [6] developed a multi-axis prosthetic ankle joint to mimic as many degree-of-freedom as possible of a human ankle. However, this prosthesis still can store very little elastic energy compared to the usage of large deflection compliant beams. Phan., et al. [7] designed a rehabilitation device for human mobility-assisting using a compliant constant-torque mechanism (CTM). Their design exploited the benefit of compliant mechanisms by simplifying the device with elastic curve beams in a monolithic form. No sensorized control systems and actuators are required in assisting the rehabilitation of human joint with this device. POM material was proposed to used for the design and the prototypes of their devices can be fabricated using CNC milling process. The monolithic 
nature of this CTM would be commercial feasibility if the manufacturing cost concern are addressed. Mass production with injection molding can resolve this issue instead of using $\mathrm{CNC}$ milling for every single piece.

Light and durable materials such as carbon fibre/epoxy composites are popular and ideal materials for orthopaedic prosthetics. Generally, fibre composites can be made by either long or short fibre production processes. While short fibre composites production relies on injection molding machines, composites from carbon-fibre fabric can be made manually with simple manufacturing process. The key characteristic of composite prosthetics is the fact that they can be custom-made to match the height, weight, and muscular structure of the individual. This paper proposes the design and fabrication process of a new carbon-fibre prosthesis (CFP). The shape and stiffness of the device are optimized to facilitate the mobility and functionality of lower-limb amputees. Finite element analyses are carried out to evaluate the biomechanical behaviours of the design obtained by an optimization procedure. Prototypes of the device are fabricated using carbon/polyester composites. Experiments are performed to demonstrate the effectiveness of the device.

\section{Materials and Methods \\ Operational principle}

Ideal prostheses are supposed to assist patients in achieving natural gait motion which includes: dorsiflexion, eversion, impact absorption, energy return and ankle torsion. The fully compliant prosthetic ankle of Pham., et al. [8] fulfilled above requirements. However custom-made product to meet individual needs is a matter of cost. Figure 1 illustrates the schematic of the prosthesis in this paper which consists of a base plate, a keel plate and a rubber wedge. The rubber wedge is attached to the base plate but it is separated to the keel plate. Both base plate and keel plate are designed as simple as possible to adapt to the carbon fiber composites fabrication process. The combination of these parts has the function of shock absorption, foot flexion and strain energy storage and release.

A typical gait cycle of a sound foot is composed of two phases: stance phase and swing phase [8]. A proper prosthesis should have the capacity to store energy in order to replicate the motion of an intact foot. The design of a passive prosthetic foot mostly focused on the stance phase. When the foot hits the ground, owning to the flexibility of the heel or the rubber wedge in this design it absorbs the shock induced by the terrain (heel-strike). This passive device continues to deflect and stores elastic energy when the patient stands completely on one leg (foot-flat). A dorsiflexion in the subdivision of stance phase releases part of the energy in the heel and transfers partly to the keel. The foot accumulates mechanical energy in elastic structures in early to mid-stance and reverts it to the amputees to propel their body forward in late stance (toe-off).

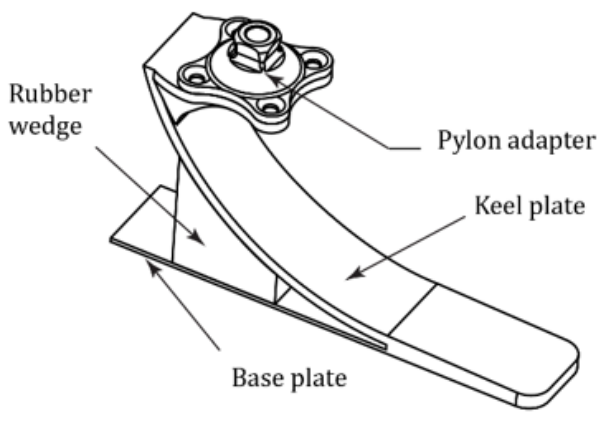

Figure 1: Schematics of the CFP.

\section{Design formulation}

An appropriate prosthetic design should ensure for a natural gait. It is expected to have the capability of reducing the impact due to the reaction force, enabling ankle rotation flexibility during movement and allowing walking on undulating and rough terrains. The CFP in this research includes two flexural beams and an elastic rubber wedge. The key component of the design is a cubic Bezier curved beam functioned as the keel whereas its curvature and thickness will define the global stiffness of the prosthesis. This stiffness could be modulated to accommodate individual needs by varying the shape of the curve beam or the corresponding Bezier control points in an optimization procedure. Generally, an $n^{\text {th }}$ order Bézier curve is determined by $(\mathrm{n}+1)$ control points $K_{i}$ as shown in figure 2:

$P(v)=\sum_{i=0}^{n} K_{i} B_{i, n}(v)$

$B_{i, n}(v)=C_{n}^{i} v^{i}(1-v)^{n-i}(0 \leq v \leq 1)$

$C_{n}^{i}=\frac{n !}{i !(n-i) !} i=0, \ldots, n$

where $B_{i, n}(v)$ is Berstein polynomial, $v$ is independent parameter, $P(v)$ and $K_{i}$ denotes position vectors of points on the curve and control points, respectively. 


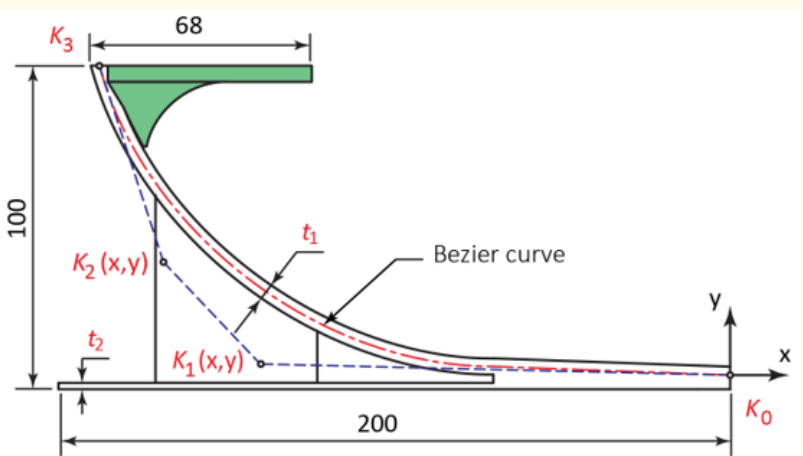

Figure 2: Design parameters.

The fixed positions of two points $K_{0}$ and $K_{3}$ are constrained in a design space $(100 \times 200) \mathrm{mm}$. Coordinates of the remaining two control points $K_{1}(x, y)$ and $K_{2}(x, y)$ are design variables. The thickness $t_{1}$ and $t_{2}$ of the keel beam and the base beam are also design variables. During the calculation process, three loading cases in figure 3 are applied to the CFP which resemble the three phases in a walking cycle: the heel strike, the foot flat and the toe-off, respectively.

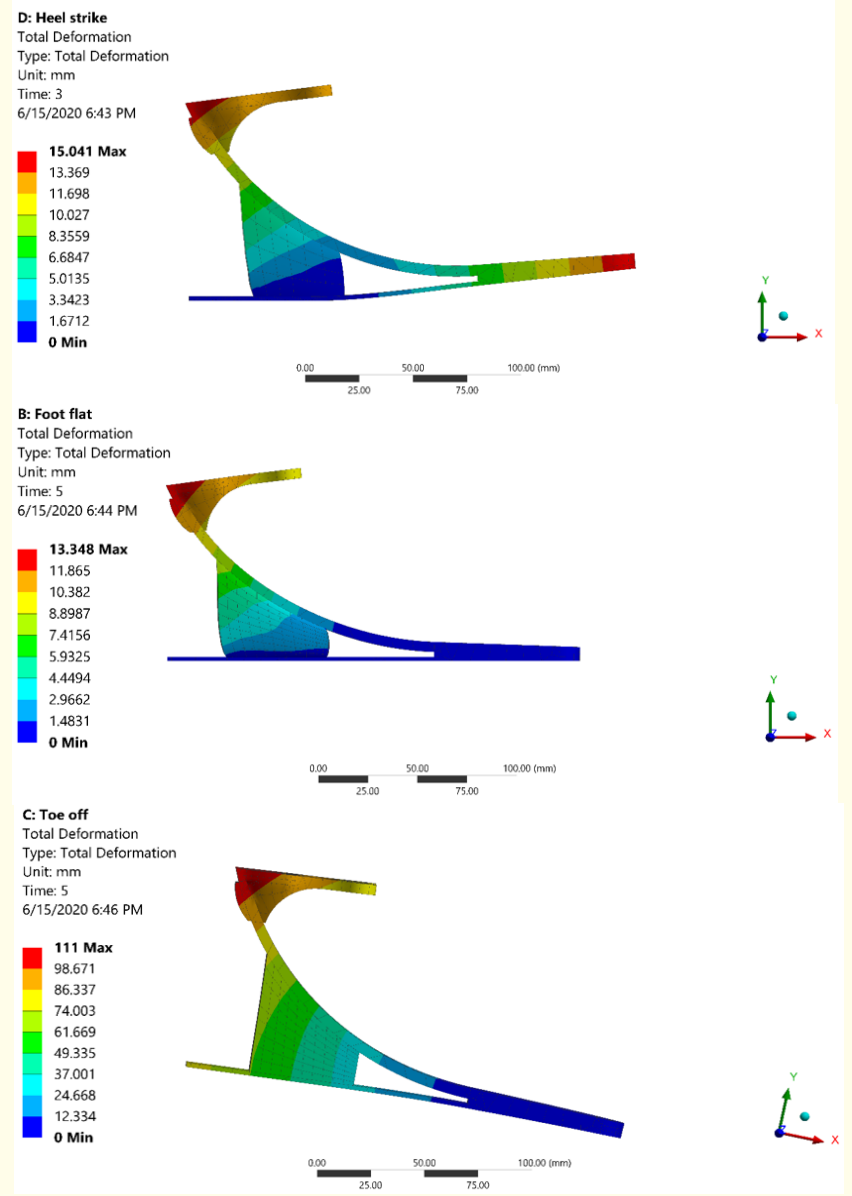

Figure 3: Three loading phases during the walking cycle: (a) Heel strike, (b) Foot flat, (c) Toe-off.
In this research, dry carbon-fiber fabric and polyester resin are used to make the composite material by using hand layup method. Firstly, the resin is mixed with catalyst and hardener additive and stirred thoroughly by hand. This compound is then vacuumed to eliminate completely all remaining bubbles. Then dry fabrics are placed into the mold layer by layer with resin mixture addition following each layer. The expected thickness of the product will define the number of fabric layers required. The final curing step with pressure applied via a vacuum bagging is maintained at room temperature for 45 minutes (Figure 4a). A fabricated carbon/polyester composite sample is shown in the inset of figure $4 \mathrm{a}$.

To determine the flexural properties of the fiber-reinforced plastic composite, test specimens are prepared following the British Standard BS EN ISO 14125:1998. The dimensions of specimens for three-point bending test are $(100 \times 15 \times 2) \mathrm{mm}$. A tensile testing machine Instron 3367 (USA) is used to implement the experiment as shown in figure 4b. The Young's modulus, Poisson's ratio and tensile strength of the specimens are found as $16.0 \mathrm{GPa}, 0.39$ and $243 \mathrm{MPa}$, respectively.

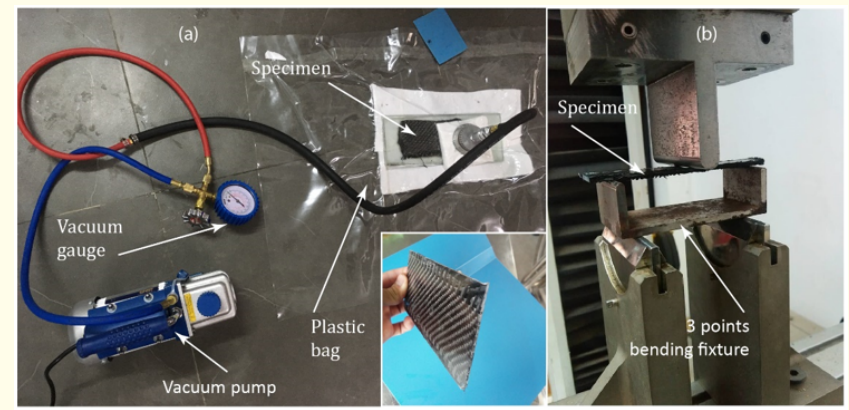

Figure 4: Fabricated sample preparation (a) and the three-point bending test experiment (b)

\section{Optimization}

The deformation analysis of the CFP is a large deflection problem. Appropriate stiffness of the structure according to each phase of the gait is searched by using an optimization scheme. An effective shape optimization procedure using genetic algorithm for the design of compliant prostheses was developed in our previous researches [9]. In the optimization process, the structure, constraints on the design variables, number of generations, and size of populations are specified.

Due to the large deflection and nonlinearity problem, the relation between applied force and displacement is calculated by using finite element analysis. A commercial software ANSYS [10] is used to calculate for the deflection of the structure. In order to make the model simple and easy for the optimization, beam element is used. 
Boundary conditions are set for a person weight from $75 \mathrm{~kg}$ to 80 $\mathrm{kg} ; 1.8 \mathrm{~m}$ in height and walking with a speed of $1.25 \mathrm{~m} / \mathrm{s}$ [11]. Optimization problem for the CFP shape design is formulated in table 1. In this optimization process, the number of generations and the size of populations are set to 50 and 20, respectively. Optimum numerical results of the design are described in table 2 and the CFP's shape is plotted in figure 5 .

Table 1: Optimization formulation of the CFP.

\begin{tabular}{|c|c|}
\hline $\begin{array}{l}\text { 1. Objective: } \\
\begin{aligned} & \operatorname{Min}\left|K_{f e m}-K_{e}\right| \\
& \\
& \operatorname{Min}\left(\sigma_{\max }\right)\end{aligned}\end{array}$ & $\begin{array}{l}(4) \\
(5) \\
\end{array}$ \\
\hline $\begin{array}{l}\text { 2. Design variables: } \\
\begin{array}{l}\text { - } \\
\text { - } \quad \text { Beamtrol points: } K_{1}(x, y), K_{2}(x, y)\end{array} \\
\end{array}$ & \\
\hline $\begin{array}{l}\text { 3. Constraints: } \\
\begin{array}{l}\text { - Design space: }(200 \times 100) \mathrm{mm} \\
\text { - Beam thickness: } 2.0 \leq t_{i} \leq 8.0 \mathrm{~mm} \\
\text { - The maximum stress: } \sigma_{\max }<\sigma_{\mathrm{y}} / \mathrm{SF}\end{array}\end{array}$ & \\
\hline
\end{tabular}

Table 2: Value of optimum design variables.

\begin{tabular}{|l|c|c|c|}
\hline Variables & Value $\mathbf{( m m )}$ & Variables & Value $\mathbf{( m m )}$ \\
\hline$K_{0}(x, y)$ & $(0,0)$ & $t_{1}$ & 5.0 \\
\hline$K_{1}(x, y)$ & $(-160,0)$ & $t_{2}$ & 2.0 \\
\hline$K_{2}(x, y)$ & $(-173,42)$ & Width & 42.0 \\
\hline$K_{3}(x, y)$ & $(-195.1,95.6)$ & & \\
\hline
\end{tabular}

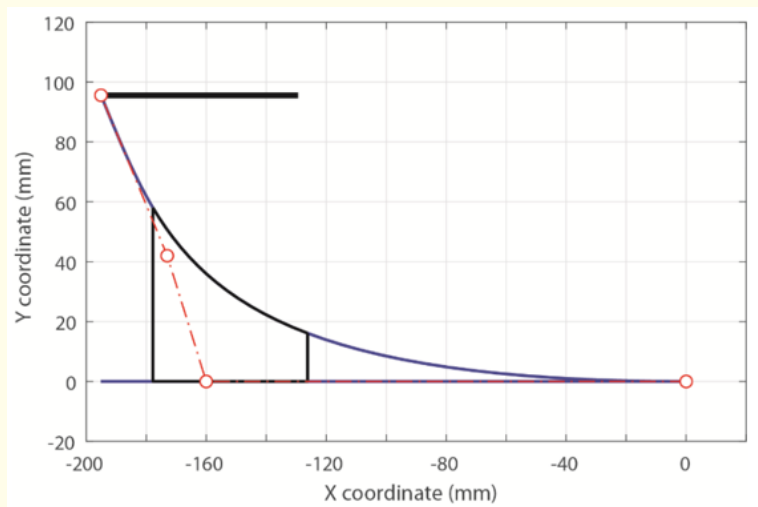

Figure 5: Optimum shape of the CFP.

\section{Fabrication and testing}

In order to realize the optimum design of the CFP for practical investigation, prototypes of the device are fabricated. Firstly, a mold whose cavities encapsulate the shape of the beams is prepared as shown in figure 6 a. The top surface is the cavity of the keel and the bottom surface is the cavity for the base plate. Following the hand layup fabrication process described in the section design formulation, a prototype is fabricated as shown in figure $6 \mathrm{~b}$. Figure $6 \mathrm{c}$ is the CFP when it is assembled with a socket for a lower-limb amputee.

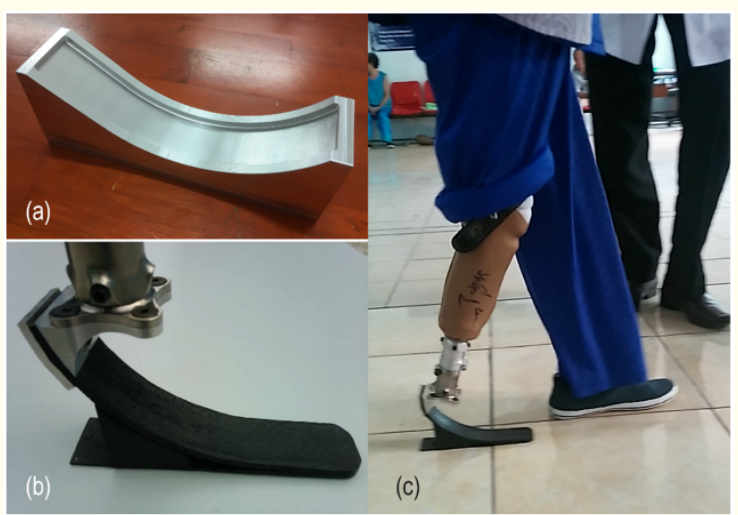

Figure 6: Fabricated mold and product.

The CFP was mechanically tested using a uniaxial tensile testing machine (Instron 3367, USA) (Figure 7). It was clamped to the base of the machine and loaded by a flat plate attached to a load cell follow the ISO 10328 standard for structural testing of lower-limb prostheses. It was axially loaded in three different configurations: heel-only contact (b), foot-flat (c) and toe-only contact (d).

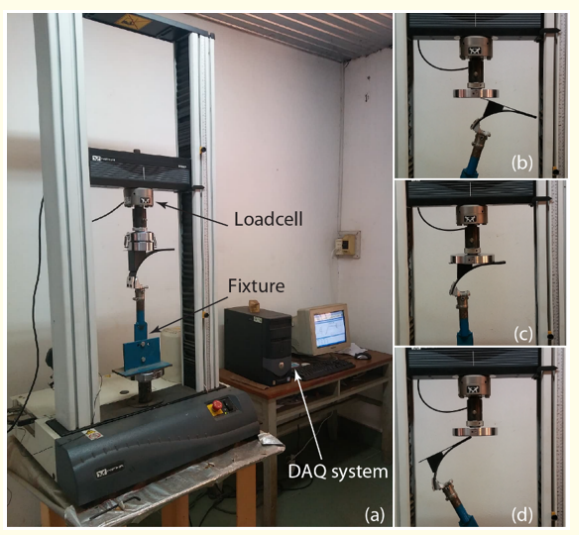

Figure 7: Mechanical testing (a) of the CFP during (b) heel-only, (c) foot-flat and (d) toe-only.

\section{Results and Discussion}

Using the experimental setup in section fabrication and testing, the stiffness of the CFP at three different loading configurations are demonstrated. Their corresponding experimental force - displacement $(f-d)$ curves are also obtained. Figure 8-10 show the measurement results for each loading configuration. The calculated stiffness is also presented in these figures for comparison. It can 
be seen that only the foot-flat loading in figure 8 shows a slightly proportionality between force and displacement with the regression stiffness of simulation and experiment are $34.1(\mathrm{~N} / \mathrm{mm})$ and $41.1(\mathrm{~N} / \mathrm{mm})$, respectively. The difference between calculated and practical results can be attributed to the uncertainties in material, geometry and loading conditions of the experiments. Any slightly change in the resin mixture as well as curing treatment during the fibre composite fabrication will affect its mechanical properties. The heel-strike and toe-off loading curves in figure 9 and 10 are completely nonlinear but they comparatively follow the simulated results. The contact of the flat plate of the testing machine with the surfaces of the prosthesis in all loading cases are not fixed, where sliding may occur, and the alignment of the load cell with the symmetry plane of the CFP may not be perfect during the experiments. In a nutshell, the results show rather good agreement between simulation and measurement.

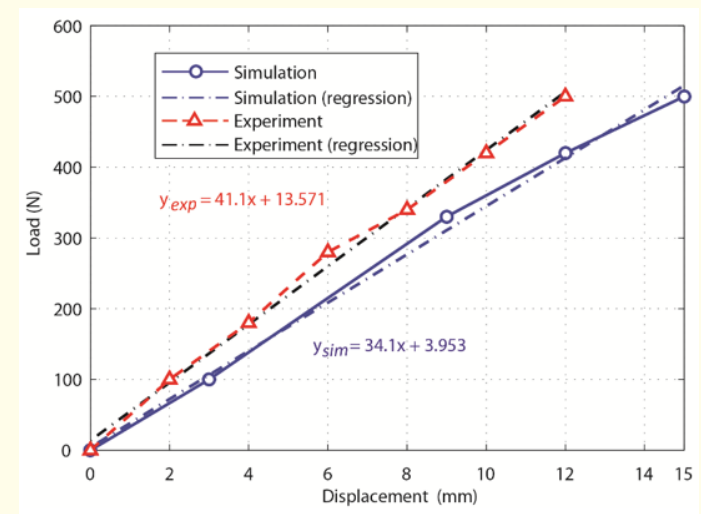

Figure 8: Force-displacement curve for foot-flat loading.

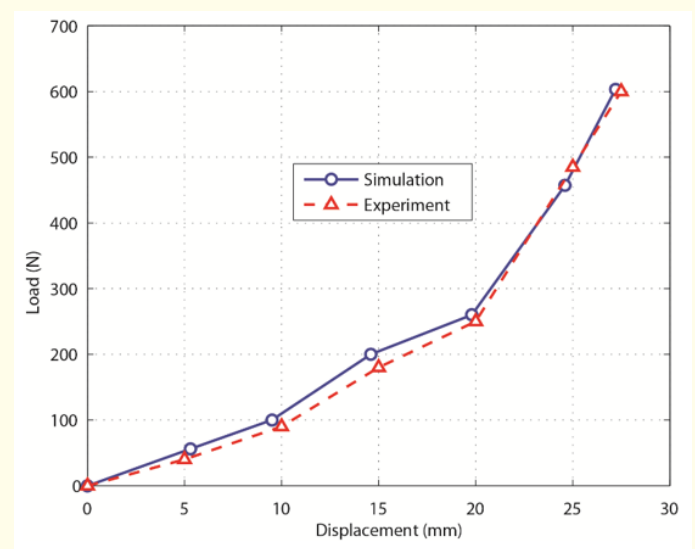

Figure 9: Force-displacement curve for heel-strike loading.

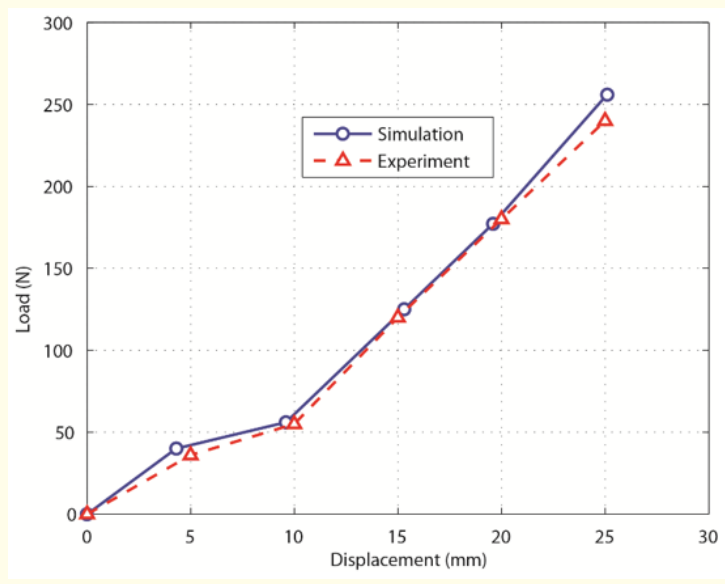

Figure 10: Force-displacement curve for toe-off loading.

\section{Conclusion}

In this research, the design methodology of a new carbon/polyester composite prosthesis has been proposed. Taking advantage of fully compliant mechanisms, without any movable joints, the use of the designed device would result in reduced wear, no need for maintenance and enhanced performance by increasing precision. In order to evaluate the effectiveness of the device, prototypes of the device are fabricated using a simple hand layup composite production process. They are also validated by experiments. The combination of choosing appropriate material and using compliant mechanisms further enhances the capability of storing elastic energy in early to mid-stance of the prosthesis and releasing it to assist the amputees in forward propulsion in late stance. The optimization methodology using in this research could be incorporated to forward dynamics simulations [12] of amputee walking and replace fixed setting component stiffness by a proper metabolic cost and join loading objective functions [13] in order to account for practical feelings of amputees.

\section{Acknowledgements}

This work belongs to the project grant (No: T2020-59TD) funded by Ho Chi Minh City University of Technology and Education, Vietnam. The authors also would like to acknowledge Mr. Huynh Van Phi M.D. and Orthopaedic and Rehabilitation Centre, Ministry of Labour - Invalids and Social Affairs, Ho Chi Minh city, Vietnam for their useful advices and assistant in testing with below-knee amputee.

\section{Conflict of Interest}

None. 


\section{Bibliography}

1. Kim H., et al. "Design and locomotion control of a hydraulic lower extremity exoskeleton for mobility augmentation". Mechatronics 46 (2017): 32-45.

2. Long Y., et al. "Development and analysis of an electrically actuated lower extremity assistive exoskeleton". Journal of Bionic Engineering 14 (2017): 272-283.

3. Torrealba RR., et al. "Design of variable impedance actuator for knee joint of a portable human gait rehabilitation exoskeleton". Mechanism and Machine Theory 116 (2017): 248-261.

4. South BJ., et al. "Manufacture of energy storage and return prosthetic feet using selective laser sintering". Journal of Biomechanical Engineering 132 (2010): Article ID 015001.

5. Hafner BJ., et al. "Energy storage and return prostheses: does patient perception correlate with biomechanical analysis?" Clinical Biomechanics 17 (2002): 325-344.

6. Lee CL., et al. "Multi-axis prosthetic ankle joint". U.S. Patent, Ohio Willow Wood Company, US 6,699,295 B2 (2004).

7. Phan TV., et al. "Design and Analysis of a Compliant ConstantTorque Mechanism for Rehabilitation Devices". In: Parinov I, Chang SH, Long B (editions). Advanced Materials. Springer Proceedings in Materials 6 (2020): 541-549.

8. Pham HT and Nguyen VK. "A monolithic flexural-based prosthetic foot for amputee". Journal of Engineering Technology and Education 9.5 (2013): 461-467.

9. Pham HT., et al. "Shape optimization and fabrication of a parametric curved-segment prosthetic foot for amputee". Journal of Science and Technologies: Technical Universities 102 (2014): 89-95.

10. Ansys ${ }^{\circledR}$ Academic Research Mechanical, Release Help System, ANSYS, Inc 18.2.

11. Torburn L., et al. "Below-knee amputee gait with dynamic elastic response prosthetic feet: A pilot study". Journal of Rehabilitation Research and Development 27 (1990): 369-384.

12. Fey NP., et al. "Altering prosthetic foot stiffness influences foot and muscle function during below-knee amputee walking: A modelling and simulation analysis". Journal of Biomechanics 46 (2013): 637-644.

13. Fey NP., et al. "Optimization of prosthetic foot stiffness to reduce metabolic cost and intact knee loading during belowknee amputee walking: A theoretical study". Journal of Biomechanical Engineering 134 (2012): 111005.

\section{Assets from publication with us}

- Prompt Acknowledgement after receiving the article

- Thorough Double blinded peer review

- Rapid Publication

- Issue of Publication Certificate

- High visibility of your Published work

Website: https://www.actascientific.com/

Submit Article: https://www.actascientific.com/submission.php Email us: editor@actascientific.com

Contact us: +919182824667

Citation: Huy-Tuan Pham., et al. "Optimization Design of a Carbon Fibre Prosthetic Foot for Amputee". Acta Scientific Orthopaedics 3.10 (2020): $16-21$. 